ENCYCLOPEDDIE Encyclopédie berbère

BERBERE $36 \mid \mathbf{2 0 1 3}$

36| Oryx - Ozoutae

\title{
Ouarsenis : Écologie du massif
}

Fatiha Abdoun

\section{(2) OpenEdition}

Journals

Édition électronique

URL : https://journals.openedition.org/encyclopedieberbere/2851

DOI : 10.4000/encyclopedieberbere.2851

ISSN : 2262-7197

\section{Éditeur}

Peeters Publishers

\section{Édition imprimée}

Date de publication : 2 juin 2013

Pagination : 5922-5928

ISBN : 978-2-7584-0194-0

ISSN : 1015-7344

\section{Référence électronique}

Fatiha Abdoun, « Ouarsenis : Écologie du massif », Encyclopédie berbère [En ligne], 36 | 2013, document 038, mis en ligne le 12 mars 2021, consulté le 17 février 2022. URL : http://journals.openedition.org/ encyclopedieberbere/2851; DOI : https://doi.org/10.4000/encyclopedieberbere.2851

Ce document a été généré automatiquement le 17 février 2022.

(c) Tous droits réservés 


\title{
Ouarsenis : Écologie du massif
}

\author{
Fatiha Abdoun
}

1 L'Ouarsenis est l'appellation actuelle d'un massif montagneux appartenant à la chaîne de l'Atlas tellien. Orientée ouest-sud-ouest à est-nord-est, cette chaîne constitue avec l'Atlas saharien l'essentiel du système orographique nord-africain s'étendant de la côte atlantique marocaine jusqu'en Tunisie. Cependant, le terme Ouarsenis désigne localement le seul pic culminant à $1985 \mathrm{~m}$ (Pic Sidi Amar) au centre de ce massif.

2 L'Ouarsenis couvre une superficie de près de $14500 \mathrm{~km}^{2}$ répartie sur 6 wilayas (Aïn Defla, Chleff, Tissemsilt, Tiaret, Médéa et Relizane). Situé entre les Monts des Béni Chougran et les monts du Titteri, entaillé par les affluents de l'oued Cheliff et limité à l'ouest par l'oued Mina, au nord par l'oued Cheliff et par la vallée de Nahr Ouassal au sud (Sari 1977). 

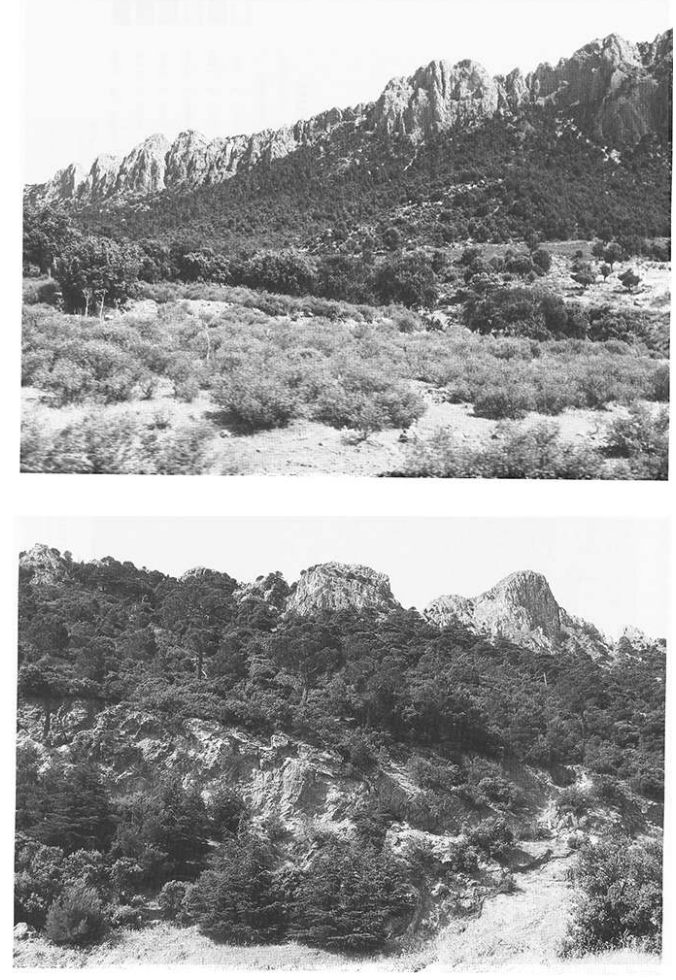

FIg. 1 \& 2 : VUES DU DJEBEL BOUCAÏD, LE VERSANT SUD (OUARSENIS 1) SUPPORTE UNE FORMATION VÉgétAle dOMINÉE PAR LE PIN D'ALEP ET CHÊNE VERT ; LE VERSANT NORD (OUARSENIS 2) EST COUVERT PAR LA CÉDRAIE DOMINANTE À PLUS DE 1200 M D'ALTITUdE

Clichés F. Abdoun, juillet 2010.
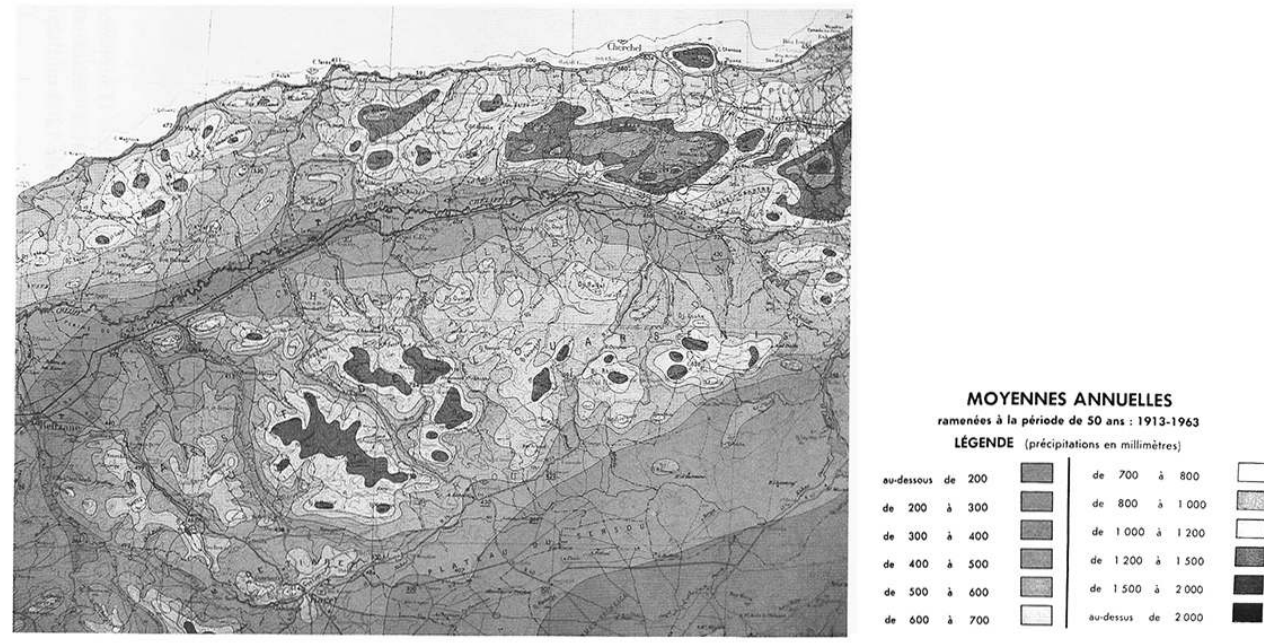

Fig. 3 : CARTE PLUVIOMÉtRIQUe de L'OUARSENIS (D'APRÈS CHAUMONT ET PAQUIN, 1971). 


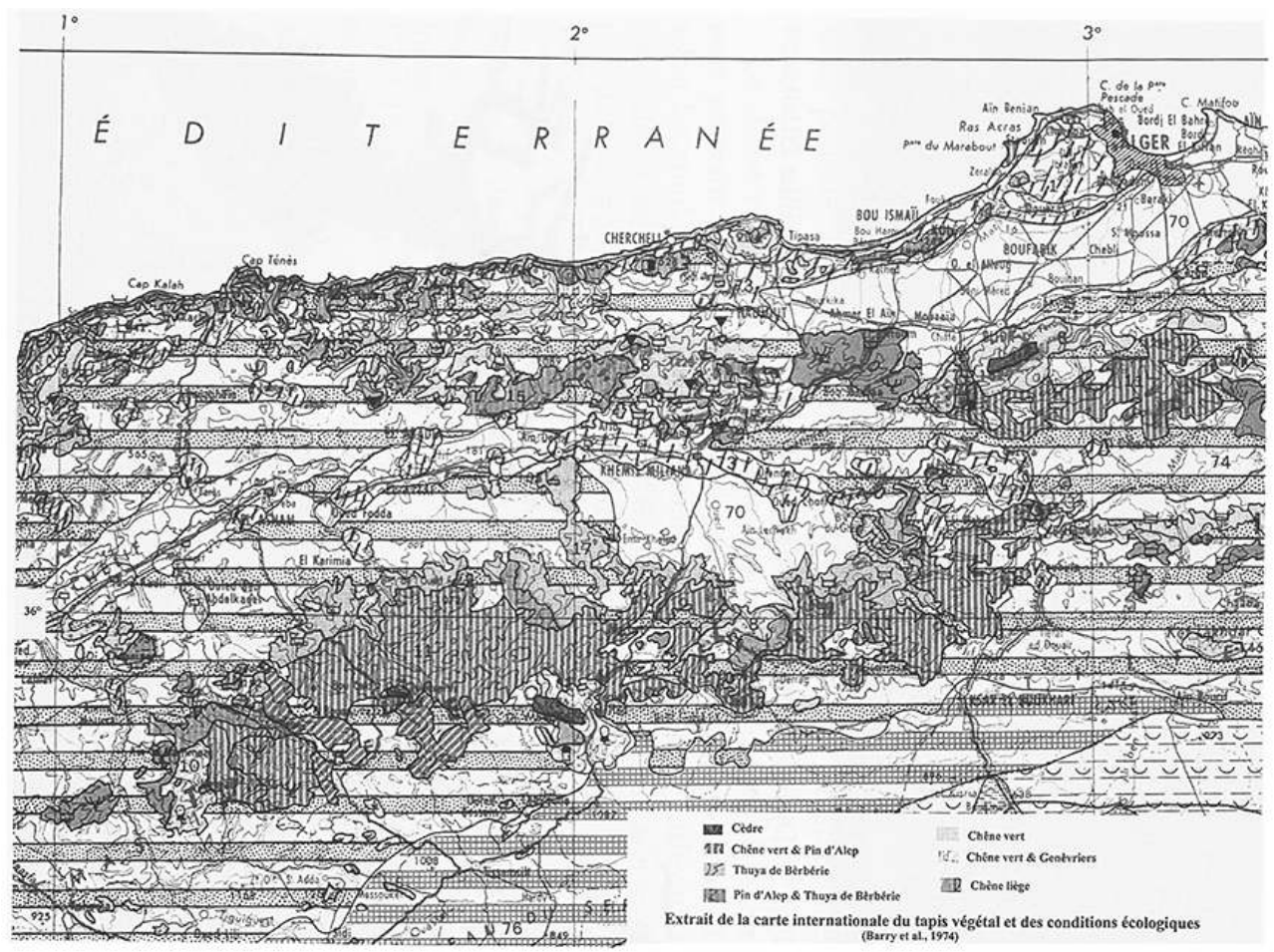

FIg. 4 : CARTE dE LA VÉgÉtATION (D'APRÈS BARRY, CELLES ET FAUREL L. 1974).

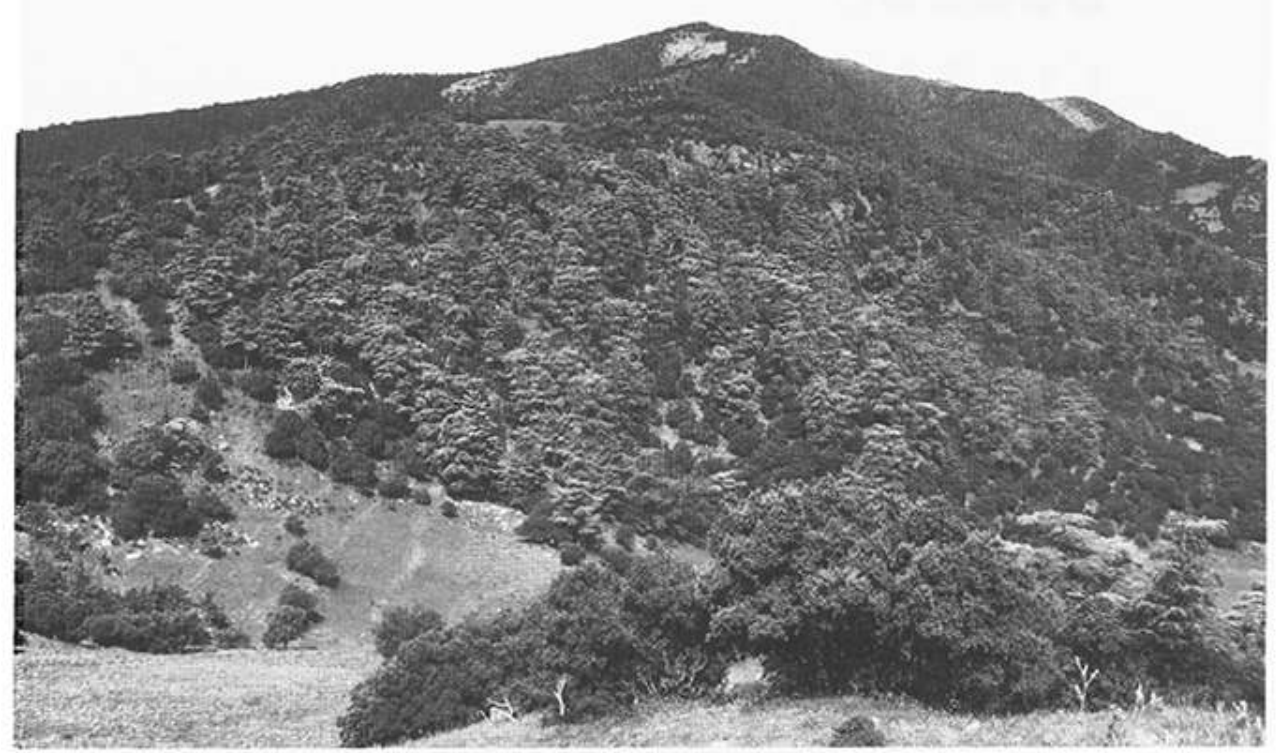

FIg. 5 : DJEBEL MEDdAD, VUE PAYSAgÈre

Cliché F. Abdoun, juillet 2010. 


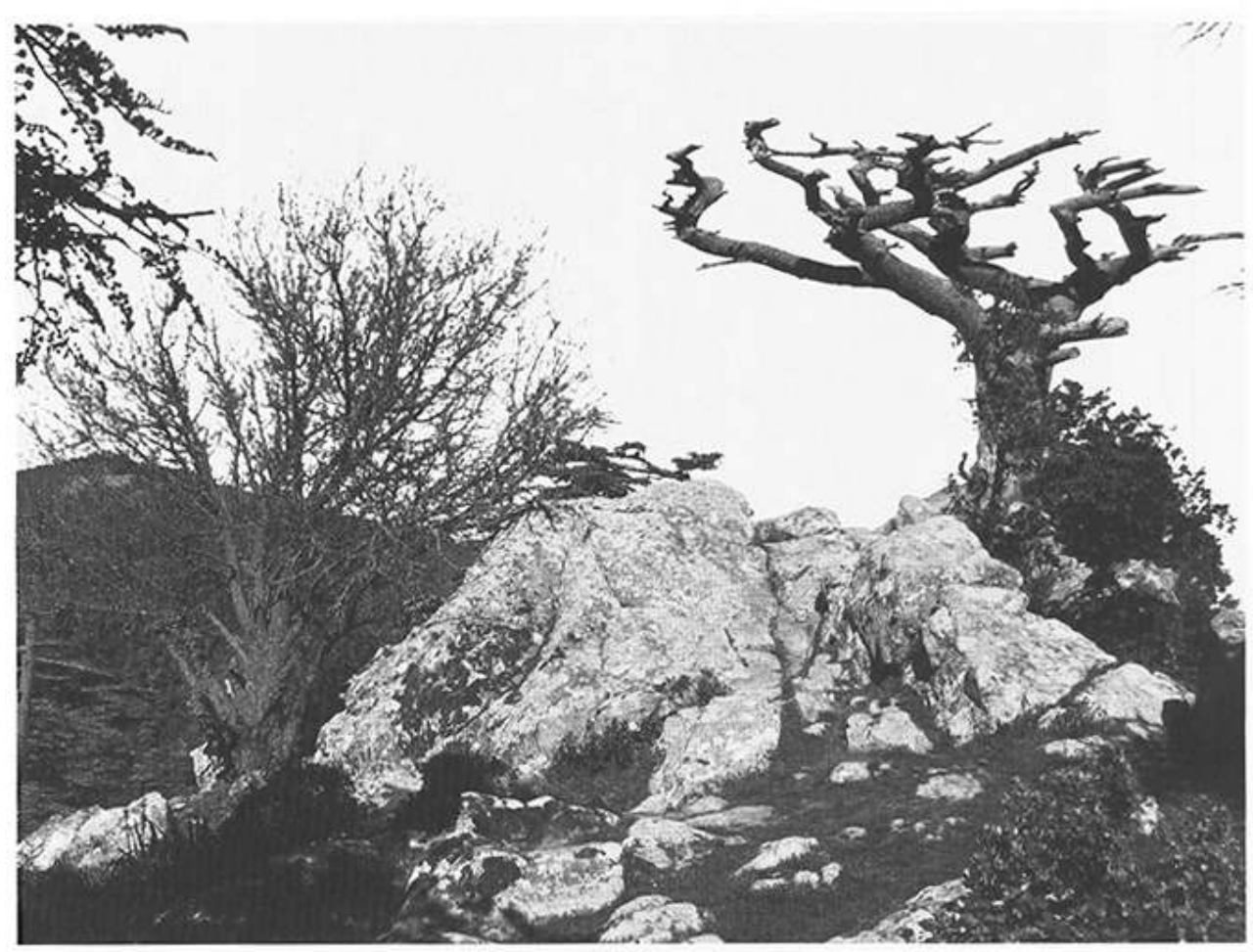

FIg. 6 : DJEBEL MEDDAD, LE PISTACHIER DE L'ATLAS, À gAUCHE, AU POINT APPELÉ PARASOL EN RÉFÉRENCE AU CÈDRE MORT, À DROITE, À 1460 M d'ALTITUDE

Cliché F. Abdoun, avril 2012.

3 Le climat de l'Ouarsenis varie du subhumide au semi-aride, il est relativement privilégié par la pluviométrie qui est supérieure à $400 \mathrm{~mm} / \mathrm{an}$ et, aux plus hautes altitudes, à $800 \mathrm{~mm} / \mathrm{an}$, quantité suffisante pour entretenir une couverture forestière. Cependant les précipitations sont irrégulières et torrentielles engendrant de fortes érosions. Les températures et les précipitations divisent l'année en deux saisons séparées par de brèves transitions printanière et automnale. Une saison hivernale qui ne dure que deux mois suivie par un été assez long de 8 à 9 mois dans les bas versants (Sari 1977.)

4 Le relief de l'Ouarsenis et son climat privilégient une diversité de paysages végétaux qui depuis les basses jusqu'aux hautes altitudes, présente des formations de l'olivierlentisque, du pin d'Alep, chêne vert, du thuya de Berbérie et des enclaves de cèdre de l'Atlas et de chêne liège.

5 Le massif est recouvert en grande partie par des futaies de pin d'Alep et des taillis de chêne vert. Le pin d'Alep est une essence liée essentiellement au climat thermoméditerranéen atténué mais supporte l'ambiance climatique mésoméditerranéenne. Son développement s'accorde avec une saison sèche pouvant atteindre 6 mois et l'optimum de son développement se situe entre 400 et $600 \mathrm{~mm} / \mathrm{an}$ (Kadik 1987).

6 Le chêne vert est le plus souvent associé au pin d'Alep dans un rapport pouvant être dominant ou dominé (Carte de Barry et al. 1974) selon les conditions environnementales à savoir, climat, anthropisation, incendies. Ces derniers favorisent le pin et, en raison de son caractère pyrophile et pyrogène, le feu s'étend grâce à lui, vite et bien (Barry et al. 1974). 
7 Le thuya, espèce endémique d'Afrique du Nord, dominant dans la partie occidentale du massif, sous forme de forêts claires ou de steppes arborées, est présent ça et là dans les parties centrale et orientale. Vestige d'un passé forestier luxuriant qui a rendu célèbre le mont Ancorarius (Ouarsenis selon Gsell 1913 ; cf. notices 039 et 040). Pendant la première moitié du $\mathrm{XX}^{\mathrm{e}}$ siècle il couvrait environ 27000 ha de la région algéroOuarsenisienne (Boudy 1955).

8 Le cèdre, espèce phare, est actuellement présent sous forme de deux petits lambeaux aux Djebels Meddad (un des noms berbères du cèdre de l'Atlas ${ }^{1}$ ) et l'Ouarsenis. Ces deux cédraies de l'ouest algérien sont relativement bien conservées (Sarmoum 2008) par rapport aux cédraies des Aurès où le dépérissement prend des proportions alarmantes (Bentouati \& Bariteau 2006).

9 Les cédraies auraient occupé une aire beaucoup plus vaste durant l'Holocène selon les données palynologiques (Salamani 1991) et seraient en voie de disparition en Algérie selon des modèles de simulation qui prédisent le déplacement de son aire géographique vers des latitudes nord-européennes à la faveur du réchauffement climatique (Cheddadi 2005). Sera-t-il remplacé par le pistachier de l'Atlas qui se contente encore du versant sud du Djebel Meddad et qui, sur le piémont de son versant nord, tend à frôler la cime des cèdres?

\section{BIBLIOGRAPHIE}

BARRY J.-P., CELLES J.C., FAUREL L., 1974 - Carte internationale du tapis végétal et des conditions écologiques, Feuilles d'Alger au 1/1000000, Alger, Société d'Histoire Naturelle d'Afrique du Nord.

BARRY J.-P., CELLES J.-C., FAUREL L., 1974 - Notice de Carte internationale du tapis végétal et des conditions écologiques, Feuilles d'Alger au 1/1000000, Alger, ONRS, CRBT.

BENTOUATI A., BARITEAU M., 2006 - «Réflexion sur le dépérissement du cèdre de l'Atlas dans les Aurès (Algérie) », Forêt méditerranéenne, XXVII, p. 317-322.

BOUDY P., 1955 - Economie forestière Nord-africaine, t. IV : description forestière de l'Algérie et de la Tunisie, Pairs, Edit. Larose, $483 \mathrm{p}$.

CHAUMONT M., PAQUIN C., 1971 - Carte pluviométrique de l'Algérie au 1/500 000, Société d'Histoire Naturelle de l'Afrique du Nord.

CHEDDADI R., 2005 - « Impact du climat sur quelques espèces méditerranéennes : du passé au futur ", Colloque sur la Biodiversité et changements globaux, Inst. Franç. Biodiv., p. 91-96.

KADIK B., 1987 - Contribution à l'étude du Pin d'Alep (Pinus halepensis Mill.) en Algérie :écologie, dendrométrie, morphologie, Alger, OPU, $581 \mathrm{p}$.

SALAMANI M., 1991 - « Premières données paléophytogéographiques du cèdre de l'Atlas (Cedrus atlantica) dans la région de Grande Kabylie (NE Algérie) », Palynosciences, 2, p. 147-155.

SARI D., 1977 - L'homme et l'érosion dans l'Ouarsenis (Algérie), Alger, SNED, 624 p. 
SARMOUM M., 2008 - Impact du climat sur le dépérissement du cèdre de l'Atlas (Cedrus atlantica Manetti). Diagnostic dendroécologique et climatique de la cédraie de Theniet El Had (Wilaya de Tissemsilt), Mém.

Magister, USTHB, Alger, 98 p.

\section{NOTES}

1. Ce terme (avec le sens de cèdre) est donné par E. Destaing dans son Dictionnaire français-berbère (dialecte des Beni-Snous) (Paris, 1914), pour les Matmata, voisins de l'Ouarsenis, sous la forme lmeddad (avec l'article arabe) ; R. Basset, dans son Etude sur la zénatia de l'Ouarsenis (Paris 1895, p. 81), donne également thameddad ([Өaməddad], forme curieuse au plan de la morphologie berbère, avec une probable variante [məddad], par amuissement et chute fréquente dans les parlers «zénètes» de la marque initiale du féminin ( $t a>\theta a,>h a-,>\varnothing$; cf. notice 038c). Cette forme ne semble pas connue ailleurs (les autres dialectes ont: ingəl (kabyle); idgəl itgəl (tamazight du Moyen-Atlas). Le Dictionnaire pratique arabe [dialectal]-français de Beaussier (Alger, 1887/1931, p. 923) donne : meddad = « cèdre de l'Atlas », que l'on retrouve également dans Trabut (Répertoire des noms indigènes des plantes... dans le Nord de l'Afrique, Alger, 1935), avec d'autres dénominations berbères et arabes. On ne le trouve cependant pas dans les dictionnaires d'arabe classique, ce qui confirme l'origine berbère de cette dénomination. NDLR.

\section{INDEX}

Mots-clés : Algérie, Botanique, Ecologie, Géographie 